山्यRANÇAISE

$>\mathrm{DE}$

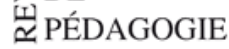

\section{Revue française de pédagogie}

Recherches en éducation

199 | avril-mai-juin 2017

L'histoire des disciplines : un champ de recherche en mutation

\title{
L'enseignement des langues étrangères sous la Troisième République : des disciplines en prise avec les relations internationales
}

The Teaching of Modern Languages in France from the 1870s to 1940: School

Subjects at the Heart of International Relations

Jérémie Dubois

\section{OpenEdition}

Journals

Édition électronique

URL : https://journals.openedition.org/rfp/6005

DOI : 10.4000/rfp.6005

ISSN : 2105-2913

Éditeur

ENS Éditions

Édition imprimée

Date de publication : 30 juin 2017

Pagination : 23-37

ISBN : 979-10-362-0108-0

ISSN : 0556-7807

Référence électronique

Jérémie Dubois, «L'enseignement des langues étrangères sous la Troisième République : des disciplines en prise avec les relations internationales », Revue française de pédagogie [En ligne], 199 | avril-mai-juin 2017, mis en ligne le 30 juin 2020, consulté le 06 janvier 2022. URL : http:// journals.openedition.org/rfp/6005; DOI : https://doi.org/10.4000/rfp.6005 


\title{
L'enseignement des langues étrangères sous la Troisième République : des disciplines en prise avec les relations internationales
}

\author{
Jérémie Dubois
}

\begin{abstract}
L'histoire de l'enseignement des langues étrangères en France met en lumière l'ambiguïté du rapport de la France au monde durant les années 1870 à 1940. La volonté de renforcer et de diversifier les langues enseignées en France, en particulier autour des années 1900-1902, s'accompagne d'efforts récurrents pour instrumentaliser l'enseignement des langues étrangères afin de peser dans les relations internationales. Les enseignants des différentes langues, comme l'anglais, l'allemand, l'italien, l'espagnol, I'arabe ou le russe, sont à la fois des enjeux et des acteurs dans les rapports entre la France et d'autres États.
\end{abstract}

Mots-clés (TESE) : enseignement des langues étrangères, transfert d'enseignants, éducation interculturelle, offre en langues étrangères

\section{Introduction}

Le champ de l'histoire de l'enseignement des langues vivantes en France à l'époque contemporaine a connu grâce aux travaux pionniers des années 1990 un important renouveau. Des recherches consacrées à I'histoire de l'enseignement de l'allemand ont débouché sur la construction d'un nouvel objet historiographique, portant sur la position et le statut des maîtres de langues comme intermédiaires culturels entre deux pays (Espagne, Lagier \& Werner, 1991). La place de l'histoire des enseignants de langue dans l'élaboration de l'ap- proche des «transferts culturels» par Michel Espagne et Michael Werner a donné une impulsion scientifique et une légitimité nouvelle à l'étude du milieu et de l'action de ces professeurs, en incitant à rechercher des liens entre l'espace des salles de classe et les enjeux sociaux, culturels, diplomatiques et politiques (Espagne $\&$ Werner, 1994). Ainsi, parallèlement aux travaux explorant I'histoire de la didactique des langues (Puren, 1988; Germain, 1993), la transmission des langues en contexte scolaire a de plus en plus été examinée sous 
l'angle des liens entre histoire de l'éducation et histoire de la circulation transnationale des personnes et des savoirs. Un renversement de perspective s'est ainsi opéré, puisque les enseignants de langues, structurellement dominés dans l'appareil éducatif français au cours du XIX siècle (Rogers, 2006, p. 138), ont été érigés en échelle pertinente d'analyse pour penser des dynamiques - mais aussi des tensions ou des blocages dont ils peuvent être les acteurs ou les enjeux.

Une importante bibliographie s'est ainsi développée. Aux études visant à explorer un milieu enseignant spécifique, celui des professeurs d'allemand en lycée en France sous le Second Empire (Brethomé, 2004), répondent des approches interrogeant pour les décennies ultérieures les symétries et les asymétries entre les enjeux de l'enseignement de l'allemand en France et ceux du français en Allemagne (Mombert, 2001, 2014). Le numéro consacré en 2005 par la revue Histoire de l'éducation à l'enseignement de l'allemand du XIXe au $\mathrm{XXI}^{\mathrm{e}}$ siècle témoigne bien de la place durablement singulière de l'histoire de l'allemand en France dans I'historiographie des disciplines linguistiques (Mombert, 2005). Les recherches de Sébastien Rival sur les échanges d'assistants entre France et Allemagne avant 1940 ont récemment éclairé l'arrière-plan diplomatique des enjeux pédagogiques (Rival, 2012). L'une des caractéristiques de ces travaux est d'avoir contribué à susciter des recherches sur la transmission d'autres langues, ce qui conduit à revenir désormais sur l'affirmation liminaire de l'important ouvrage de Monique Mombert selon laquelle, autour de 1900, "aucune autre matière scolaire ne dépendait comme l'allemand de représentations qui n'étaient pas liées à l'univers pédagogique » (Mombert, 2001, p. 8). Des travaux portant sur d'autres idiomes et ne se focalisant pas uniquement sur les processus techniques de transmission amènent à suggérer l'existence d'une forme de singularité plurielle, commune aux différentes disciplines linguistiques et portant sur leurs interactions plus ou moins étroites avec les cultures politiques et les milieux sociaux auxquelles elles sont liées, tant à propos de l'espagnol (Niño Rodriguez, 1988; Delaunay, 1994, 2010; Bennassar, 1994; Herreras, 2008) que de l'anglais (Pouly, 2009, 2012), du russe (Guichard, 1999; Cœuré, 2004)', de l'arabe (Messaoudi, 2015), du chinois (Ber-

1 Voir également la thèse en cours de Sandra Dominique sur la langue et la culture russes dans l'enseignement supérieur français, thèse préparée à l'université Paris-Diderot sous la direction de gère \& Pino, 1995 ; Daniel, Grangé, Zhuxiang et al., 2015) ou de l'italien (David, 1984; Dubois, 2015a). Les examiner simultanément, dans leurs caractéristiques singulières et dans leurs points communs, amène à mettre au jour des débats, des négociations et des systèmes de représentation dans lesquels elles sont imbriquées. L'étude du destin des langues scolaires éclaire ainsi le rapport de la France à d'autres cultures et à d'autres États ${ }^{2}$.

Le choix d'étudier dans cet article les années 1870 à 1940 tient d'abord à l'intérêt - peut-être contreintuitif de prime abord - d'une analyse de la diffusion des langues étrangères durant une période où, en France, de multiples initiatives visent à affirmer l'universalité de la langue française, notamment par le biais de l'Alliance française (Chaubet, 2006). Parallèlement, I'État républicain entretient un rapport complexe aux cultures et aux langues régionales : s'il vise certes à répandre l'usage scolaire du français, il ne mène pas systématiquement une politique d'exclusion des parlers régionaux des élèves (Chanet, 1996; Michel, 2016). Dans ce contexte, l'étude des modalités de «l'inscription scolaire» (Pouly, 2009) des langues étrangères vivantes dans le système éducatif français participe aussi à l'écriture d'une histoire pédagogique et politique des autorités éducatives, actuellement en plein renouveau à l'échelle du ministère, des académies et des établissements (Loubes, 2012; Condette, 2006b, 2015). Le premier objectif de cet article est de s'interroger sur la définition heurtée, inégale et controversée de langues tierces comme «bonnes langues » scolaires, institutionnellement et socialement légitimes, dans une France cultivant une fascination collective pour sa propre langue et pour le grec et le latin (Waquet, 1998). Il s'agit également d'éclairer l'histoire des langues scolairement fortes par celle de langues qui le sont moins en saisissant les dynamiques qui rendent ce paysage moins statique qu'il n'y paraît. Sous l'effet de réformes scolaires comme celle de 1902 (Pouly, 2010; Héry, 2015) ou en raison d'événements politiques ou économiques, ces hiérarchies se reconfigurent, parfois fortement, comme en témoignent les positions fluctuantes de l'enseignement du russe, de l'italien ou de l'arabe en France durant la période.

2 Cette porosité entre les univers disciplinaires et extra-scolaires n'est pas spécifique au domaine des disciplines linguistiques ni même au seul champ des sciences humaines et sociales : elle s'observe aussi pour des matières scientifiques, comme le montrent les recherches comparées de Catherine Radtka $(2013,2018)$. 
Cette perspective amène à interroger plus largement le rôle respectif des enseignants de langues, de l'État et d'autres acteurs sociaux dans la diffusion scolaire des langues vivantes. L'extension ou la diminution de la place accordée aux langues vivantes en général et à chacune d'entre elles en particulier dépend-elle de choix volontaristes effectués par l'État et son personnel politique ou représente-t-elle une réponse à des d'initiatives et demandes issues du monde enseignant, de la société civile ou de puissances étrangères? Les gouvernements se montrent-ils en l'espèce plutôt stratèges ou suiveurs? Les volontés d'acteurs étrangers sont-elles prises en considération lors des arbitrages?

Cet article vise dès lors à contribuer à l'étude du rapport des Français aux langues étrangères sous la Troisième République, au prisme de trois caractéristiques du monde des enseignants de langues. En premier lieu, il s'agit d'éclairer les causes et les limites d'une relative montée en puissance des langues étrangères dans l'enseignement secondaire français durant la période, pour mesurer la part de facteurs liés à des enjeux internes au monde scolaire et ceux qui ressortissent à d'autres causes, liées aux échanges culturels et diplomatiques internationaux. Après avoir insisté sur les dynamiques à l'œuvre durant la période, nous explorerons un aspect du lien entre pédagogie et circulations internationales à travers les débats sur l'intervention en France d'acteurs étrangers dans l'enseignement des langues. La question du recours à des «locuteurs natifs», c'est-à-dire à des enseignants de nationalités autres que française, diffusant en France leur langue maternelle, suscite souvent un intérêt ambivalent, comme si la langue étrangère ne pouvait se transmettre que par le filtre d'un professeur français, garant d'une «renationalisation » de l'idiome étranger à destination des écoliers de France. L'objectif est ici d'analyser les dimensions sociale, politique et pédagogique de ce protectionnisme disciplinaire, qui vise à préserver le marché de I'enseignement français des langues de l'intervention d'acteurs extérieurs. Enfin, nous nuancerons ce tableau d'un monde enseignant parfois prudent face à l'influence pédagogique étrangère directe en montrant que, durant l'entre-deux-guerres, des professeurs de langues témoignent d'un engagement à la fois professionnel et personnel destiné à mettre leur métier au service d'un dialogue pacifique entre les peuples d'Europe. Alors que les professeurs de langues en France peuvent être l'objet de tentative d'instrumentalisation par des puissances étrangères, ils n'en cherchent pas moins aussi parfois à jouer un rôle d'acteur dans les relations internationales ${ }^{3}$.

Ces trois facettes du métier d'enseignant de langue en France sous la Troisième République sont explorées ici à partir de trois types de sources : des publications de l'époque, associatives ou issues de la presse d'information générale; des fonds conservés aux Archives nationales et dans des archives départementales, retraçant notamment les parcours des premiers lecteurs de langues étrangères dans les universités en France et leurs interactions avec le monde de l'enseignement secondaire ${ }^{4}$; et enfin la correspondance de I'Association des professeurs de langues vivantes (APLV) avec le ministère de I'Instruction publique dans I'entre-deux-guerres ${ }^{5}$.

\section{Les enjeux idéologiques et internationaux de l'enseignement des langues vivantes}

Du lendemain de la défaite de 1870 aux années 1930, des acteurs aux profils très divers se mobilisent pour que l'apprentissage des langues vivantes soit rendu moins «secondaire» dans les établissements scolaires français. L'idée que la France pourrait mieux « tenir son rang» parmi les nations du globe en développant la compétence de ses élèves en langues étrangères est défendue de façon récurrente. Elle forme le ciment de I'alliance de circonstance entre les promoteurs des différentes langues vivantes en France, par ailleurs rivaux entre eux. À cette lecture répond à l'inverse un discours contre les langues vivantes et leurs réformes, dénonçant en celles-ci des modèles venus d'ailleurs,

3 Nous explorons ici à l'échelle de différentes langues étrangères la portée d'un modèle d'analyse croisant les enjeux disciplinaires et les relations politiques et diplomatiques. Nous avons développé cette approche à propos de l'enseignement de l'italien en France. Voir Dubois, 2015a.

4 Nous nous appuyons sur des dossiers issus des Archives nationales (site de Pierrefitte), en particulier sur les dossiers AN/F/17/13245 (lecteurs de langues étrangères dans les facultés des lettres, 19071919) et le dossier AN/20010498/3. Nous avons aussi consulté les dossiers individuels des lecteurs dont nous avons pu retrouver les traces aux archives départementales de la Gironde (Bordeaux). Voir par exemple les dossiers de «Mr Gassmann, lecteur d'allemand à la faculté des lettres, 1906-1907», carton 1774 W 49; "M. Giesen Joseph, lecteur d'allemand à la faculté des lettres, 6 avril 1909 - cesse mars 1910 », carton 1774 W 52; "M. Joachim, lecteur d'allemand à la faculté des lettres, 18 novembre 1908 », 1774 W 61 ; «Mr Schaub », 1774 W 103, archives départementales de la Gironde, Bordeaux.

5 Voir le dossier «Association des professeurs de langues vivantes 1928-1934», Archives nationales, AN/70/AJ/28. 
dont la promotion risquerait d'accélérer et d'entériner le déclassement du pays dans le monde, dans la mesure où chaque effort entrepris en faveur des langues étrangères vaudrait reconnaissance du fait que le français ne suffit plus pour échanger à échelle du monde. Ce débat rebondit dans les colonnes du grand quotidien d'information généraliste Le Siècle, en automne $1913^{6}$. Le journal présente la controverse entre un opposant aux réformes des langues vivantes ${ }^{7}$ et le groupe des professeurs de langues qui anime la revue Les Langues modernes $^{8}$. Le journal s'engage clairement contre P. Roques, qui contestait les nouvelles méthodes d'enseignement et l'importance donnée aux langues étrangères en France dans la Revue universitaire. Il y écrivait notamment: "c'est tenir une gageure contre la nature » que de vouloir «donner à de petits Français, vivant en France, une âme allemande pendant quatre ou cinq heures par semaine ${ }^{9}$ ». L'influent quotidien parisien réfute l'idée de Roques selon laquelle «la base de l'enseignement est et ne peut être dans nos lycées que la langue française», thèse que le journal condense en soulignant que pour ce dernier, «le professeur de langue vivante doit être le collaborateur du professeur de français ${ }^{10} »$. Ces différents types d'approches, témoignant d'un rapport parfois patriotique voire nationaliste à la langue étrangère, forment le substrat dans lequel s'est déployée la compétition disciplinaire, en lien avec les aires culturelles et nationales auxquelles chacune des langues étrangères concernées est liée.

\section{La position dominée de l'enseignement des langues vivantes en France}

Durant les premières années de la III République, l'héritage de Victor Duruy, ministre de I'Instruction publique

6 Voir l'article «Pour la méthode directe» du journal Le Siècle du 4 septembre 1913, p.6, (non signé). Cet extrait a été repéré et consulté grâce au nouveau site des archives de presse de la BnF, $<$ www.retronews.fr $>$.

7 Voir P. Roques, «Dix ans de méthode directe», Revue universitaire, $\mathrm{n}^{\circ} 7,1913$, p. 108-115. Sur le retentissement de cet article polémique dans la presse pédagogique, voir Puren, 1988.

8 Cette revue est l'émanation de l'Association des professeurs de langues vivantes. Ses collections ont été entièrement numérisées sur la bibliothèque numérique de la $\mathrm{BnF}$, <gallica.bnf.fr $>$, en partenariat avec l'association.

9 P. Roques, cité dans l'article signé L. M., «Dix ans de méthode directe», Les Langues modernes : bulletin mensuel de la Société des professeurs de langues vivantes de l'enseignement public, août 1913 $\left(11^{e}\right.$ année, $\left.n^{\circ} 8\right)$ qui en donne un compte rendu très critique, p. 442. 10 La formule figure à la fois dans la recension citée des Langues de Napoléon III entre 1863 et 1869, est ambivalent. Duruy a voulu encourager l'apprentissage des langues vivantes; il obtient en 1865 la création de la filière de «l'enseignement secondaire spécial», dans laquelle les langues vivantes ont une place conséquente. Toutefois, l'image de cette nouvelle filière est fragilisée par l'absence en son sein des humanités latines et grecques (Geslot, 2009, p. 197). La légitimité des langues vivantes à former les jeunes esprits reste contestée, le latin demeurant un «instrument d'autorité » au sein de la société (Waquet, 1998, p. 288). L'ascendant des langues anciennes au sein de la hiérarchie des disciplines scolaires est donc paradoxalement conforté par les réformes de la fin du Second Empire.

Parallèlement, les langues vivantes gagnent en audience dans le système éducatif français à la faveur de la construction d'un enseignement public secondaire féminin. Le ministre Victor Duruy s'inspire pour l'élaborer en 1867 du modèle de l'enseignement spécial, en privilégiant une formation littéraire générale sans latin, que les langues vivantes complètent. La promotion des langues vivantes est là encore ambiguë, puisque ces matières étendent leur présence scolaire au sein de filières construites pour ne pas déboucher sur le diplôme du baccalauréat. Les réformes républicaines portées par Camille Sée en 1880 accentuent le paradoxe : les nouveaux établissements secondaires féminins suscitent des créations de postes d'enseignantes en langues vivantes mais leur position dans le système éducatif reste peu valorisée. À un moment où le latin et le grec restent des "bastions masculins» (Rogers, 2006, p.135), les places fortes des langues vivantes apparaissent comme des filières dominées, bien qu'elles soient en développement.

\section{Les nouveaux défenseurs des langues vivantes}

Plusieurs facteurs liés aux relations internationales concourent à un renforcement des langues vivantes étrangères sous la Troisième République. L'impulsion en est paradoxale, puisque ce projet pédagogique a eu pour point de départ une réaction collective face à une défaite militaire. La débâcle des armées françaises dans la guerre franco-prussienne de 1870 a été propice à une mise en discussion globale du système éducatif en France, de nombreuses voix appelant à des transformations radicales au nom du relèvement du pays. Sur le plan de la société civile, l'intense activité déployée sous l'impulsion des frères Siegfried par la 
Société pour la propagation des langues étrangères en France témoigne de l'intérêt des milieux industriels et patronaux pour la question (Pouly, 2007). Cette association, qui réunit des professeurs de langues et des membres des élites économiques, cherche à convaincre les responsables politiques de la nécessité d'améliorer les compétences linguistiques des jeunes Français pour favoriser les exportations et le rayonnement international de la France.

Les motivations diplomatiques et stratégiques, nationales ou nationalistes sont aussi très présentes chez certains défenseurs des langues, qui voient dans la diffusion en France de certains idiomes une arme véritable dans les rivalités entre nations. La classe de langue vivante est dès lors parfois conçue comme un lieu où se joue un aspect des relations entre la France et des peuples et États étrangers. Cette perspective est directement celle du normalien et agrégé de lettres Charles Dejob, qui fut le principal artisan de la création d'une agrégation d'italien en France en 1900 et le premier maître de conférences d'italien à la Sorbonne (Dubois, 2015a). Ce dernier mobilise un vaste réseau de professeurs de lycées et d'universitaires italiens et français au sein de la Société d'études italiennes qu'il crée en 1894 pour promouvoir le développement de l'apprentissage de la langue italienne en France. Son objectif est loin d'être seulement culturel et linguistique. Comme il s'en explique dans un ouvrage qu'il a réservé à une publication posthume (Dejob, 1919), son projet voit le jour dans un contexte où, depuis 1882, I'Italie avait rejoint l'alliance germano-autrichienne. L'aggravation des tensions franco-italiennes lors du massacre d'ouvriers italiens par des travailleurs français à Aigues-Mortes en $1893^{11}$ pousse Dejob à agir pour améliorer la connaissance et l'estime de l'Italie contemporaine en France. II espère susciter en retour une inflexion de l'opinion transalpine en faveur de la France. Son intention est de contribuer à sortir la France de son isolement diplomatique en obtenant la bienveillance de personnalités italiennes susceptibles d'être sensibles aux initiatives de diffusion de leur langue dans l'enseignement secondaire et supérieur en France. Son objectif central est bien la Revanche qui permettrait d'obtenir le retour à la France des provinces perdues d'Alsace-Moselle : la langue italienne enseignée dans les collèges et lycées français doit ainsi servir à convaincre les Italiens de soutenir diplomati-

11 Voir sur ces événements Noiriel, 2010. quement et à terme militairement la France dans sa volonté de récupérer Strasbourg. Charles Dejob a ainsi constamment relié son effort pour structurer un enseignement secondaire de l'italien en France avec l'objectif de contribuer à un renversement d'alliance. À un moment où la politique menée en Italie par Crispi est largement hostile à la France, Dejob et certains des professeurs d'italien ou des italophiles qu'il fédère autour de lui en France semblent bien faire de la politique par d'autres moyens. En revanche, il ne cherche pas à fédérer au sein de sa Société d'études italiennes des personnes issues de la vaste immigration italienne en France : il préfère rechercher le soutien des élites intellectuelles d'Italie.

Dès lors, l'ambition visant à diffuser les langues étrangères vivantes par souci d'ouverture culturelle, dans une approche idéaliste au nom du cosmopolitisme ou de la fraternité des peuples suivant un modèle hérité des années 1848, n'a pas forcément une grande place au tournant du siècle. Certains universitaires semblent néanmoins y croire, comme Julien Luchaire, maître de conférences à l'université à Lyon, qui analyse l'essor des langues étrangères comme un «signe des temps pacifiques» et estime en 1902 que les cours de littératures étrangères ont vocation à être des «foyers de sympathie pour chacun des peuples auxquels ils sont respectivement consacrés» (Luchaire, 1902, p. 260-261).

\section{Le destin croisé des langues vivantes, un enjeu du rapport de la France au monde?}

Les années de la Troisième République représentent un moment clé dans la diversification et l'extension de l'importance des langues vivantes dans l'appareil éducatif français, même si les objectifs ne sont pas toujours atteints. Inspirée par les résultats d'une grande enquête parlementaire, la réforme du baccalauréat en 1902 donne beaucoup plus de poids aux langues vivantes: les filières modernes avec langues vivantes doivent désormais être placées au même niveau que les filières classiques avec latin et grec (Isambert-Jamati, 1969; Prost, 2008; Pouly, 2010). Le corps des professeurs de langues étrangères s'agrandit et se diversifie alors qu'ouvrent de nouvelles agrégations de langues, en 1900 pour l'espagnol et l'italien et en 1906 pour l'arabe ${ }^{12}$.

12 Sur l'agrégation comme indicateur clé dans l'institutionnalisation d'une discipline dans l'enseignement secondaire en France, voir Chervel, 1993 et Verneuil, 2005. 
Ces concours s'ajoutent aux agrégations d'anglais et d'allemand qui existent déjà depuis 1848. Les agrégations de langues vivantes sont les premières à devenir des concours mixtes, ouverts indifféremment aux femmes et aux hommes à partir de 1883. Rebecca Rogers y voit un signe de la moindre reconnaissance sociale des agrégations de langues, situées «au bas de l'échelle du concours ». À la différence d'autres agrégations, celles de langues sont longtemps ouvertes à des candidats dépourvus de licence (Rogers, 2006, p. 142; voir aussi Efthymiou, 2003).

Au sein des langues vivantes, le poids de l'allemand et de l'anglais sous la Troisième République doit beaucoup au fait que ces langues ont été les premières à bénéficier d'agrégations disciplinaires ${ }^{13}$. La domination des anglicistes et des germanistes sur le corps des professeurs agrégés de langues est écrasante. Entre 1880 et 1939, 857 agrégés sont reçus en anglais et 653 en allemand. Par comparaison, le nombre total des lauréats reçus avant 1939 pour les agrégations d'espagnol (113), d'italien (105) et d'arabe (26) peut sembler dérisoire $^{14}$. En réalité, les quelques postes ouverts annuellement au concours s'accompagnent de la structuration de filières universitaires spécifiques dont les effets vont se faire sentir dans la formation des maîtres de l'enseignement secondaire, y compris lorsque ceux-ci ne réussissent pas le concours de l'agrégation. Surtout, ces nouveaux concours vont favoriser la création de charges spécifiques d'inspection des langues vivantes, désormais assurées par des universitaires spécialisés qui vont s'attacher à développer chacune des langues dont ils sont les représentants. Le contraste est net avec la situation qui prévalait vers 1890 , lorsque les enseignants d'espagnol ou d'italien étaient évalués par des inspecteurs généraux des langues vivantes généralistes, qui étaient en fait souvent des anglicistes et des germanistes, comme Adolphe Bossert et Émile Chasles. Pour l'arabe, c'est seulement après la Première Guerre mondiale qu'un arabisant, William Marçais, «maître incontesté des études arabes en France» (Messaoudi, 2011, p. 156), est chargé d'une mission d'inspection des établissements du second degré.

Face aux langues les mieux établies, l'allemand et l'anglais, les destins scolaires de l'italien, de l'espagnol,

13 En allemand et en anglais, le concours a connu une longue interruption entre 1852 et 1864 (Chervel, 2015). Bien que le texte de 1848 ait porté en théorie aussi sur l'italien et l'espagnol, aucune session d'agrégation n'est ouverte pour ces langues avant 1900. de l'arabe et du russe sont assez différents. Ces quatre langues ont bénéficié au tournant du siècle d'un soutien institutionnel accru, mais dont les effets furent différents selon les langues. L'ouverture d'une agrégation en 1900 pour l'italien et l'espagnol a été précédée d'une longue mobilisation des partisans de ces deux langues, en particulier autour de l'hispaniste Ernest Mérimée, depuis la faculté des lettres de Toulouse, et de l'italianisant Henri Hauvette, depuis celle de Grenoble. Ces derniers avaient obtenu la création de licences d'espagnol et d'italien dans leurs établissements respectifs, qui en ont eu brièvement le monopole en France à la fin du XIX $X^{\mathrm{e}}$ siècle. Ce sont principalement leurs élèves, tout juste diplômés des premières licences d'espagnol et d'italien, qui adressent en 1897 une pétition au ministre de l'Instruction publique pour obtenir l'ouverture d'une agrégation des langues méridionales ${ }^{15}$. Les lauréats de la licence signent généralement la pétition en ajoutant qu'ils occupent déjà une fonction dans l'enseignement secondaire. L'enjeu de l'agrégation est essentiel pour les nouveaux licenciés de ces deux langues méridionales, car ce concours est alors le seul titre permettant d'obtenir la garantie d'un poste en lycée. Il existe bien depuis 1841 des certificats d'aptitude à l'enseignement pour l'italien et pour l'espagnol, mais ceux-ci sont en réalités considérés comme de simples «brevets», qui ne donnent pas directement accès à un poste d'enseignant du secondaire, et qui ne permettent d'y exercer, dans le meilleur des cas, que comme chargé de cours en lycée, c'est-à-dire dans une position moins reconnue que la chaire de professeur. La licence de langue vivante représente un autre mode de qualification, mais elle ne donne pas non plus accès à un métier de façon assurée. Ainsi, en termes de construction disciplinaire et d'orientation professionnelle des étudiants, la question de l'existence ou non d'une agrégation s'avère un facteur crucial d'attractivité ou non d'une langue vivante pour les futurs enseignants. La formation et la carrière de Pierre Rouède le montrent parfaitement : ce dernier étudie le russe durant trois ans, sous la conduite du professeur Paul Boyer à l'École des langues orientales vivantes ${ }^{16}$. Il passe la moitié de l'année 1901 en Russie ${ }^{17}$. En juin 1904, il sollicite un poste de professeur de russe avec l'appui de

15 «À propos du projet d'agrégation des langues méridionales», Revue universitaire, $\mathrm{n}^{\circ} 6,1897-2, \mathrm{p} .479-480$ (non signé).

16 Lettre de Pierre Rouède au vice-recteur de l'académie de Paris, reçue le 7 juin 1904, dossier personnel de Pierre Rouède, académie de Paris, AJ/16/1463.

17 lbid. 
ses maîtres ${ }^{18}$. L'absence d'une agrégation pour la langue russe l'a certainement poussé à privilégier l'italien, matière dans laquelle il obtient une bourse d'agrégation à la Sorbonne en $1904^{19}$ avant d'être reçu à l'agrégation d'italien en 1905 et de s'éloigner ainsi du russe. Un jeu de vases communicants s'observe ainsi entre des disciplines dotées du concours débouchant sur une carrière valorisée dans l'enseignement secondaire et les autres. À cette vague de nouvelles agrégations de langues, le russe fait en effet exception. II n'existe pas d'agrégé de russe en France avant 1948 (Chervel, 2015). Benjamin Guichard a mis au jour, pour la période 18921948, une "succession de tentatives avortées d'introduction du russe » dans l'enseignement secondaire en France (Guichard, 1999, p. 119). II montre que la volonté diplomatique de sceller l'alliance militaire franco-russe est directement à l'origine des tentatives d'organisation de cours de russe dans les lycées de Paris, Saint-Omer et Lons-le-Saunier à partir de la rentrée 1892 avant que cette expérience ne tourne court en 1903, le développement du russe en France s'organisant dès lors principalement à l'École des langues orientales (Guichard, 1999, p. 130). C'est précisément autour de cette période que Pierre Rouède renonce au russe pour l'italien, la trajectoire individuelle reflétant ici des mouvements à la fois pédagogiques et diplomatiques.

Alors qu'il existe depuis 1841 des certificats d'aptitude à l'enseignement pour l'italien et pour l'espagnol, il n'en existait pas pour l'arabe : celui-ci est créé par le même arrêté qui a institué le 23 juillet 1906 une agrégation pour cette langue (Messaoudi, 2013, p. 99). Comme pour l'italien, I'inscription dans l'agenda ministériel de l'ouverture de ces nouveaux concours est davantage le fait d'enseignants ayant d'abord enseigné les lettres classiques que le fruit d'une forte mobilisation en ce sens de spécialistes de langues vivantes déjà mieux reconnues, comme l'allemand ou l'anglais. Ainsi, un ancien professeur de lettres classiques devenu professeur d'arabe, Joseph Desparmet, contribue à la réorganisation de la discipline en tant que secrétaire d'une commission instituée à cet effet, avant de devenir l'un des deux lauréats de la première session de l'agrégation d'arabe en 1907 (Messaoudi, 2008, p.48; Messaoudi, 2013, p.99).

18 lbid., lettre de recommandation de l'administrateur de l'école des langues orientales vivantes au vice-recteur de l'académie de Paris, 27 juin 1904.

19 État des services, dossier personnel Pierre Rouède, AN/F/17/24975.
Dans l'ensemble, même à l'issue de ces années propices à des avancées, le nombre des agrégés de langues vivantes reste faible par rapport à celui des agrégés de lettres, jugés notamment sur leurs compétences en latin et grec : entre 1880 et 1939, les nouveaux agrégés de lettres sont près de 2000 , soit plus que l'ensemble des agrégés de langues vivantes pour la période (Chervel, 2015). Dans ce contexte, comme leurs collègues d'autres disciplines (Cardon-Quint, d'Enfert \& Picard, 2014²0), les enseignants de langues cherchent à se grouper et créent à partir de 1902 la Société des professeurs de langues vivantes de l'enseignement public, transformée en 1912 en Association des professeurs de langues vivantes de l'enseignement public (APLV). Les enseignants qui y participent ne sont pas tous agrégés, mais ceux qui le sont occupent les responsabilités les plus importantes, qui contribuent à les désigner comme interlocuteurs des instances officielles. Beaucoup d'enseignants de langue pratiquent la multi-affiliation associative, en adhérant à la fois à I'APLV et à une structure spécifique de promotion de la langue dont ils sont spécialistes, comme l'Association des italianisants du Sud-Est (Dubois, 2015b).

Dans l'ensemble, la valeur sociale de la compétence en langue vivante augmente au cours de la période (Pouly, 2012). Au total, I'hétérogénéité du groupe des professeurs de langues vers 1900 reste très forte, au point que l'on pourrait dire que, sociologiquement, les professeurs de langues vivantes n'existent pas réellement, tant les contrastes internes sont accusés. Les nouvelles agrégations ont néanmoins contribué à intégrer dans l'appareil éducatif des enseignants de langues longtemps «extra-scolaires».

\section{Les ambivalences de la place des étrangers dans l'enseignement des langues vivantes}

Durant toute la période, il faut posséder la nationalité française pour être fonctionnaire en France. Les étrangers ne peuvent prétendre à une chaire de "professeur de lycée», réservée aux agrégés. En ce sens, si la création des agrégations d'espagnol et d'italien en 1900 est positive pour l'institutionnalisation de ces disciplines, elle a des conséquences négatives pour les enseignants étrangers qui intervenaient dans des

20 Voir aussi l'article de Kévin Dubos sur la Société des professeurs d'histoire-géographie dans le présent dossier. 
établissements français avec des statuts certes peu favorables, mais parfois dans la longue durée ${ }^{21}$. Ils sont appelés à être remplacés par des agrégés français.

\section{Lecteurs et assistants de langues : entre innovation et marginalisation}

Alors que l'on dispose d'importantes études sur les professeurs agrégés des lycées (Verneuil, 2005) et sur l'insertion des élites universitaires de la Troisième République dans des dynamiques internationales (Charle, 1994; Tronchet, 2016), les enseignants étrangers en France sont restés dans l'ombre, à l'exception de travaux récents sur les assistants d'allemand (Rival, 2012). L'histoire des lecteurs d'université en France peut contribuer à éclairer le fonctionnement de l'enseignement secondaire, tant les interactions entre les deux univers sont fréquentes et révélatrices de hiérarchies et de blocages. Les sources archivistiques sont souvent brèves et stéréotypées car les lecteurs n'ont guère intéressé les producteurs d'archives, dans la mesure où il n'était pas prévu qu'ils effectuent une carrière complète en France. Là où les dossiers personnels des fonctionnaires français aux Archives nationales sont très complets dans la série $F / 17$, du fait de la nécessité de prévoir pour eux le versement de pensions de retraite, les informations sur les lecteurs sont significativement dispersées et moins individualisées : elles figurent notamment dans des séries thématiques des Archives nationales ${ }^{22}$, dans des fonds des archives départementales ${ }^{23}$ ou encore dans des archives étrangères ${ }^{24}$. Les lecteurs d'université cumulent souvent leur charge avec celle d'assistant en lycée. Cette double appartenance, systématique à l'université de Bordeaux au début du siècle, est déplorée par le doyen de la faculté des lettres, Georges Radet ${ }^{25}$. Le rattachement à ces deux univers ne favorise pas leur intégration. Ces «locuteurs natifs» sont chargés de transmettre leur langue plutôt que de l'enseigner. II est

21 Voir par exemple les enseignements dispensés par Joseph Bellochio au collège de Menton de 1865 à 1890 . Né en 1828 près de Mantoue, il exerce à temps partiel dans l'établissement, $n$ 'intervenant que pour trois classes. Dossier personnel J. Bellochio, AN/F/17/22737.

22 Voir en particulier le dossier AN/F/17/13245.

23 Ces dossiers sont souvent significativement minces, comme celui de Kroneberg, lecteur d'allemand à Bordeaux. Archives départementales de la Gironde, dossier Kroneberg, 1774 W 63.

24 Voir par exemple les dossiers de la série Archivio Scuole des archives du ministère italien des Affaires étrangères, à Rome. prévu qu'ils encadrent des exercices pratiques, sous le contrôle de l'enseignant français.

C'est dans des universités situées dans des régions proches de l'Allemagne, à Nancy et à Dijon, que le modèle des «lecteurs de langues étrangères » a commencé à être mis en place en France, à partir de $1901^{26}$. Le rôle d'avant-garde joué sur ce point par des universités de province contrebalance la vision d'une centralisation pédagogique française selon laquelle les impulsions relèveraient surtout d'initiatives parisiennes. À l'inverse, elle suggère en creux le statut longtemps marginal de la question. Cette tentative d'innovation pédagogique est, aux yeux de ceux qui la soutiennent, directement associée au modèle allemand $^{27}$. Le système s'étend progressivement. Alors que les premières initiatives sont financées sur des fonds propres des universités, la loi du 30 janvier 1907 instaure un «crédit spécial » pour l'emploi des lecteurs de langues étrangères dans les facultés des lettres, sous le ministère $d^{\prime}$ Aristide Briand ${ }^{28}$. L'État central a ainsi plutôt un rôle de "suiveur», entérinant et étoffant a posteriori des expérimentations locales auxquelles il a longtemps marqué un intérêt limité. Des sources d'archives suggèrent une relative méconnaissance des données concernant les lecteurs au sein de l'administration centrale, comme en témoigne une succession de tableaux manuscrits, d'abord extrêmement lacunaires ${ }^{29}$, visant à synthétiser vers 1907 la présence de lecteurs de différentes langues dans les universités françaises. Le musée pédagogique de Paris sert d'intermédiaire entre les universités françaises et les candidats étrangers ${ }^{30}$; il administre également les départs à l'étranger de candidats français à un poste de ce type.

26 La délibération du conseil de l'université de Nancy du 28 février 1907 se réfère à celle du 28 janvier 1901 décidant la création d'un poste de lecteur d'allemand, AN/F/17/13245.

27 En 1900, au congrès international de l'enseignement supérieur, Henri Hauvette avait plaidé pour la présence dans les universités françaises d'un "organe nouveau, quelque chose comme les "lecteurs" des universités allemandes, c'est-à-dire un étranger, un Italien qui fasse parler les étudiants dans sa langue » (Dubois, 2015a, p. 353). 28 Couverture du dossier AN/F/17/13245.

29 Voir par exemple le grand tableau manuscrit «Lecteurs de langues étrangères» ne comportant que très peu d'informations (non daté, pochette 1906-1907). Ces tableaux, visant à présenter, pour chaque langue, les noms des lecteurs et les universités dans lesquelles ils interviennent, s'étoffent au fil des versions successives, à mesure que les informations sont recueillies. Dossier AN/F/17/13245. 30 Le recteur de l'académie de Rennes indique ainsi en 1909 que les lecteurs d'allemand et d'anglais exerçant à la faculté en 19081909 ont été «désignés» par le musée pédagogique. Lettre du recteur de Rennes au ministre de l'Instruction publique, 6 février 1909, AN/F/17/13245. 


\section{La volonté récurrente de contourner les locuteurs natifs au profit d'acteurs nationaux}

À la différence des fonctionnaires français, les intervenants étrangers ne touchent pas un «traitement », mais des «indemnités». Celles-ci ont beau être extrêmement réduites, tout se passe comme si cet argent versé à des étrangers posait problème en ce qu'il en priverait des acteurs français. À Nancy, en 1907, lorsque l'État se propose de contribuer par des crédits centraux à la prise en charge de lecteurs étrangers, il n'est pas envisagé de les rétribuer davantage : le recteur Adam propose de réorienter aussitôt les fonds dégagés de façon à mieux rémunérer un enseignant français. Là encore, la source littérale est expressive : «le peu qui nous redeviendrait disponible pourrait servir à rétribuer un peu mieux notre pauvre Anglade ${ }^{31}$ qui se morfond ici avec ses 3.600 [francs] ${ }^{32} »$. Le pronom possessif manifeste bien à l'inverse l'extériorité des lecteurs étrangers.

Plusieurs sources témoignent du souhait récurrent des acteurs locaux de substituer aux lecteurs étrangers des fonctionnaires français en poste en lycée. En 1908, c'est ainsi un professeur agrégé d'allemand au lycée de Caen, qui détient donc la nationalité française, M. Koessler, qui occupe la fonction de lecteur à l'université de cette ville. L'année suivante, le ministère, à qui le nom du professeur de lycée a été à nouveau proposé, demande au recteur de Caen de « vouloir bien rechercher si, dans [son] ressort ou en dehors, ne se trouverait pas une personne de nationalité allemande à qui cet emploi pourrait être confié $»^{33}$. II ajoute que « pour cet enseignement spécial, il a toujours été prévu que soient recrutés des lecteurs d'origine étrangère ». Le doyen de la faculté des lettres répond qu'il serait difficile de trouver un enseignant allemand pour le poste, étant donné que le lycée de Caen ne prend plus d'assistant allemand (" on n'en a eu que des ennuis » ${ }^{34}$ ). II met en avant la naissance à Strasbourg et la formation effectuée en Allemagne de Koessler qu'il propose de maintenir en poste, avant de porter l'argument à ses yeux décisif : agrégé d'allemand, il rend de grands services dans la préparation du concours.

31 Sur Anglade, voir Espagne, 1993, p. 366.

32 Lettre manuscrite du recteur de Nancy, Adam, au directeur de l'enseignement supérieur, 4 mars 1907, AN/F/17/13245.

33 Lettre du directeur de l'enseignement supérieur au recteur de Caen, 23 novembre 1909, AN/F/17/13245.

34 Lettre du doyen de la faculté des lettres de Caen au recteur de Caen, 25 novembre 1909, AN/F/17/13245
Dans l'ensemble, une sorte de hiérarchie des altérités semble exister en la matière : au lecteur allemand, I'université française semble préférer, lorsqu'elle en a l'occasion, le lecteur suisse, comme si celui-ci incarnait une position intermédiaire, moins "étrangère» que le ressortissant de la puissance allemande. Ainsi, à l'université de Montpellier, c'est M. Gross, "sujet suisse», ayant effectué des études supérieures de droit en Russie et en Allemagne, qui donne des «conférences pratiques d'allemand $»^{35}$. Cette tendance à éviter dans la mesure du possible des intervenants issus des puissances centrales devient une injonction durant la Première Guerre mondiale, lorsqu'une note d'octobre 1914 intime aux recteurs l'ordre de s'abstenir de nommer des lecteurs allemands ou autrichiens et d'écarter ceux qui seraient en poste ${ }^{36}$. À Bordeaux, le service du lecteur est continué par un réfugié lorrain, professeur à l'école pratique de commerce et d'industrie, M. Becker ${ }^{37}$. Après la guerre, la tendance des acteurs locaux à éviter l'intervention d'enseignants étrangers se poursuit : en 1924, le ministre refuse ainsi la demande de l'université de Paris visant à ce que les lecteurs de langues étrangères «conservent le traitement qu'ils avaient comme professeurs de lycée ou de collège»; le ministre souligne que ces enseignants ne lui paraissent pas les mieux à même d'exercer ces fonctions ${ }^{38}$.

Le modèle des assistants de langues et des lecteurs a bien perduré durant la Troisième République, mais les faibles rémunérations accordées à ceux-ci ont contribué à faire relativement échouer le projet du début des années 1900 visant à stimuler fortement par leur présence l'apprentissage pratique des langues. Dans l'ensemble, les conditions offertes ne suffisant pas à les retenir, lecteurs et assistants n'effectuent souvent que d'assez brefs passages dans les établissements français, à l'exception des situations où des États étrangers les prennent financièrement en charge, parfois pour des buts politiques (Dubois, 2015c). C'est le cas pour un lecteur d'italien à l'université de Nancy, financé sur des crédits de l'Italie fasciste et en poste entre 1933 et $1940^{39}$. Là encore, les enjeux du secondaire

\footnotetext{
35 Lettre du recteur de Montpellier au directeur de l'enseignement supérieur, 12 mars 1909, AN F/17/13245.

36 Minute de la direction de l'enseignement supérieur aux recteurs des facultés des lettres, 31 octobre 1914, AN F/17/13245.

37 Minute de la direction de l'enseignement supérieur au recteur de Bordeaux, 9 juillet 1915, AN F/17/13245.

38 Lettre du directeur de l'enseignement supérieur, M. Coville, au recteur de l'académie de Paris, 2 juillet 1924, AN 20010498/4.

39 Dossier personnel Alberto Caccavelli, archives historiques du
} 
et de l'enseignement universitaire sont étroitement imbriqués, puisque ces lecteurs financés par une puissance tierce interviennent parfois en lycée : à Toulouse, les cours donnés par le lecteur Salvatore Gaetani dans les lycées de jeunes filles et de garçons au début de l'année 1936 en témoignent ${ }^{40}$. Ce dernier contribue à faire émerger une demande sociale qui débouche sur la nomination à la rentrée 1936 d'un professeur agrégé d'italien au lycée de Toulouse, Paul Renucci ${ }^{41}$. Une nouvelle fois s'opère un processus de substitution d'acteurs étrangers par des enseignants français.

\section{Les professeurs de langues vivantes et les relations internationales dans les années 1920 et 1930}

La Première Guerre mondiale a accéléré I'affirmation du rôle social des professeurs de langues vivantes, beaucoup d'entre eux ayant pris part au conflit en utilisant parfois leurs compétences linguistiques dans le cadre des combats (Bourlet, 2007). L'intérêt des puissances étrangères pour l'enseignement scolaire de leur langue en France augmente, tandis que certains enseignants de langues s'investissent dans les années 1920 et 1930 à différents niveaux pour favoriser la concorde entre les peuples européens. Il est significatif que le congrès international des professeurs des langues vivantes organisé à Paris du 31 mars au 4 avril 1931 sous l'impulsion de l'APLV mette à son programme une session intitulée : "Les langues vivantes au service des relations internationales ${ }^{42}$. Certes, l'affichage de cette ambition s'inscrit dans le prolongement d'une logique collective de défense des intérêts disciplinaires. Pour autant, les archives de ce congrès témoignent que des initiatives diversifiées ont été menées dans la longue durée en direction d'élèves français et étrangers de l'enseignement secondaire.

ministère italien des Affaires étrangères, ASMAE, Rome, série Archivio Scuole, 1920-1955, pacco 95.

40 Lettre du consul d'Italie à Toulouse au ministère des Affaires étrangères italien, 18 décembre 1934, ASMAE, Rome, série Archivio Scuole, 1929-1935, b. 856, Toulouse.

41 Curriculum vitae de P. Renucci, archives privées Lucienne Portier, consultées chez Mme Lagarrigue, Courbevoie.

\section{Le rôle de l'APLV dans la construction de politiques publiques d'échanges de professeurs et d'élèves}

Organe de défense et de représentation des professeurs de langues, I'APLV s'emploie à faire prévaloir les intérêts de ses membres au ministère de l'Instruction publique puis de l'Éducation nationale. Au sein de l'organe influent qu'est le Conseil supérieur de l'instruction publique (Verneuil, 2014), la présence d'un représentant des professeurs de langues vivantes élu par ses collègues (Mombert, 2005, p. 80) contribue à donner à I'association un accès direct aux lieux où se préparent les décisions en matière de politique éducative. Parallèlement, I'APLV parvient à être influente dans une instance à vocation internationale, I'Office national des universités et écoles françaises à l'étranger (ONUEF). Cette structure, créée sous forme d'association en 1910 pour favoriser le rayonnement de l'enseignement français dans le monde ${ }^{43}$, a dans l'entre-deux-guerres des liens étroits avec I'APLV. Joseph Denis, professeur agrégé d'allemand au lycée parisien Janson-de-Sailly accède en 1926 à la présidence de l'APLV : dès l'année suivante, en 1927, il devient directeur-adjoint de I'ONUEF. II occupe cette fonction jusqu'en 1959 (Drijard, 1960, p. 9-10). Il eut ainsi un rôle clé dans les processus d'envoi d'assistants français dans des établissements secondaires étrangers. Joseph Denis est chargé par l'APLV de présenter au congrès de 1931 un rapport sur les «échanges officiels d'élèves, d'assistants et de professeurs». Son analyse est construite pour valoriser I'action de la France en matière d'expansion intellectuelle à l'étranger et pour démontrer la part que les spécialistes de l'enseignement des langues y prennent.

Détenteur d'une fonction officielle, Joseph Denis insiste sur l'intérêt national de l'œuvre qu'il anime, estimant que «le pays qui prétendrait tirer uniquement de son sol et de ses traditions nationales toute sa vie spirituelle se condamnerait bien vite à la léthargie et à la consomption $»^{44}$. La formule fait peut-être écho aux tensions internationales du début des années 1930 et à l'insistance avec laquelle certains chefs de gouvernement prônent une politique centrée sur l'exaltation des valeurs et traditions nationales. Administrateur des

43 Cette structure est documentée par les archives de la série AN/ AJ/70 aux Archives nationales, Pierrefitte; voir aussi Rival, 2012.

44 Joseph Denis, Rapport dactylographié pour le congrès de 1931, "Les langues vivantes au service des relations internationales, les échanges officiels d'élèves, d'assistants et de professeurs », p. 1, AN/70/AJ/28. 
programmes d'échange, Joseph Denis défend le rôle des langues vivantes comme un outil pour la présence de la France dans le monde et insiste sur l'intérêt des conventions d'échanges d'assistants signées avec différents pays. Sur le plan professionnel, il érige en modèle les échanges de professeurs de langues vivantes organisés avec l'Angleterre depuis 1925, dans le cadre d'un accord entre le Board of Education et I'ONUEF, mandataire du ministère de l'Éducation nationale : «les professeurs échangés sont nommés pour la durée de l'année scolaire; ils se remplacent mutuellement dans leurs chaires et continuent à recevoir de leurs établissements d'origine leur traitement habituel $^{45}$ ». II indique que, de 1925 à 1929, l'échange a concerné un enseignant de chaque pays chaque année, puis quatre en 1930-1931. La formule de Denis évoquant une « innovation heureuse, mais assez audacieuse » représente sans doute un euphémisme diplomatique pour rendre compte des difficultés pratiques du processus; il n'en espère pas moins un élargissement ultérieur de ces échanges à d'autres pays. Ainsi, même sous des formes numériquement limitées, des initiatives officielles conjointes franco-britanniques visent à faire des professeurs de langues vivantes des acteurs des échanges internationaux. Si la dimension bilatérale et institutionnelle est ici très forte, elle n'empêche pas, parallèlement, des initiatives davantage inspirées par des idéaux cosmopolites à échelle régionale, en particulier dans les régions marquées par le souvenir de la Première Guerre mondiale.

\section{Des initiatives régionales pour construire la paix par les langues}

Grâce aux travaux de I'historien Jean-François Condette, on connaît désormais bien les deux personnalités qui ont impulsé et coordonné les initiatives prises autour du rectorat et de la faculté des lettres de Lille pour favoriser la concorde internationale par des rencontres estivales entre les élèves de différents pays. Il s'agit d'Albert Châtelet, recteur de l'académie de Lille de 1924 à 1936, et de l'universitaire spécialiste de grammaire et de phiIologie françaises Charles Guerlin de Guer, qui a dirigé I'institut d'expansion universitaire lillois (Condette, 2009; Condette, 2006a, p. 177). Le projet du recteur Châtelet d'agir pour «la paix par le dialogue » (Condette, 2009, p. 238) dans une académie encore meurtrie par

45 Joseph Denis, Ibid., p. 22. la Grande Guerre a rencontré un puissant écho parmi les professeurs de langues de la région, comme en témoignent les engagements dont la trace a été conservée dans la correspondance de l'APLV. C'est ainsi en sa double qualité de professeur agrégé d'anglais et de proviseur du lycée Alexandre Ribot de Saint-Omer que Marcel Agobert signe le rapport qu'il établit pour le congrès international de 1931 à propos des cours d'été et des foyers de vacances organisés sous l'impulsion de l'université de Lille. Entre 1923 et 1927 se mettent en place des cours de français pour des élèves étrangers à Dunkerque et Calais. L'initiative vise d'abord à accueillir des élèves allemands et anglais. Elle reste proche du modèle classique des cours internationaux d'été pour étudiants, qu'elle décline simplement à un public plus jeune. L'originalité du programme s'accroît à partir de 1928, date à laquelle sont créés des «cours franco-étrangers ${ }^{46}$ pour des élèves de l'enseignement secondaire. Ces cours scolaires d'été rassemblent des élèves des lycées, collèges, et écoles primaires supérieures de France à Calais et Dunkerque, ainsi que des élèves du même âge venus de Grande-Bretagne, d'Allemagne et de Tchécoslovaquie ${ }^{47}$. L'auteur avance le chiffre de près de 600 élèves, filles et garçons, reçus au total dans ces deux centres en 1930. D’après le détail de ses chiffres, le plus important contingent est celui des élèves britanniques (330). La présence de 145 élèves français aux côtés de 90 élèves allemands et de 30 Tchécoslovaques donne à ces cours de langue estivaux une dimension de rencontre internationale. II n'est pas anodin que ces initiatives centrées sur l'apprentissage des langues aient lieu à une période où, sur le plan politique et diplomatique, les ministres français et allemand Aristide Briand et Gustav Stresemann ont recherché dans la deuxième moitié des années 1920 un rapprochement franco-allemand.

Ces rencontres sont ouvertes à des élèves ayant déjà appris une langue étrangère durant deux ans car il n'y a pas de section de débutants. Les élèves ne participent pas à ces cours à titre individuel mais au sein d'un groupe issu de leur établissement d'origine; ils demeurent sous la responsabilité de leurs professeurs, qui les accompagnent et sont hébergés gratuitement durant la durée de la session, longue, au choix, de 2 à 6 semaines, entre le 17 juillet et le 27 août. Les groupes

46 Rapport dactylographié de Marcel Agobert pour la session "Les langues vivantes au service des relations internationales", congrès de Paris, sans date, p. 2, AN/70/AJ/28.

47 Idem, p.3. 
d'élèves sont logés respectivement dans les collèges de garçons et de filles de la ville d'accueil. Concernant les règles de vie collective, le rapporteur indique que «la discipline la plus libérale est admise, à condition qu'elle ne nuise pas à la vie harmonieuse de l'ensemble ${ }^{48}$. Tout se passe comme si ces expérimentations pédagogiques et politiques fondées sur l'apprentissage des langues vivantes visaient aussi à promouvoir une éducation moins strictement autoritaire que de coutume.

Les cours de langues sont dispensés par des professeurs enseignant leur langue maternelle, les accompagnateurs s'échangeant ainsi les groupes dont ils ont la charge pour intervenir chacun devant un public étranger. Cette combinaison, dont se félicite le professeur agrégé d'anglais devenu proviseur, a beau être estivale, temporaire et expérimentale, elle n'en suscite pas moins un intérêt qui tranche avec les difficultés soulignées précédemment à acclimater en France l'intervention de locuteurs natifs dans l'enseignement secondaire. En dehors des cours, tout est fait pour que les élèves des différentes nationalités soient en contact, en vue de favoriser une «interpénétration des groupes».

Les artisans de ces cours d'été insistent sur le but politique constamment associé au souci de favoriser une meilleure connaissance pratique de la langue chez les élèves : il s'agit de "préparer des ponts pour les jeunes générations, avec cet espoir que le "pont des langues" serait un jour prochain celui des esprits et des cœurs $»^{49}$. II n'est pas anodin qu'un tel discours soit élaboré à partir d'initiatives prises dans des départements ayant connu l'occupation durant la Première Guerre mondiale. Dans le contexte du congrès international des professeurs de langue vivante de 1931, la conclusion du rapporteur déduit de ces expériences une définition renouvelée du métier, évoquant des professeurs de langues vivantes, "dont la mission la plus haute est de travailler au rapprochement des peuples en leur donnant les moyens de se connaître, de se comprendre et de s'estimer ${ }^{50}$. Ainsi s'opère au cœur de la période de l'entre-deux-guerres une forme de «retour à Babel » impliquant directement des élèves et des professeurs de l'enseignement secondaire. II ne s'agit pas ici de promouvoir une langue comme outil politique contre une autre nation, ni d'insister essentiellement sur la

48 Idem, p. 4.

49 Idem, p. 14. valeur pédagogiquement formatrice ou économiquement et socialement utile des langues, mais bien de dégager la fonction pacificatrice de cet enseignement. Si la question de la comparaison avec les langues anciennes n'est pas présente dans le rapport, cette tension lancinante demeure. En ce sens, l'engagement nourri d'un idéalisme international s'articule aussi avec un souci de distinction et d'affirmation du rôle social des langues modernes.

\section{Conclusion}

Dans l'ensemble, parmi les acteurs de la promotion des langues étrangères vivantes en France, interviennent donc des enseignants et des universitaires aux positionnements idéologiques très divers. À l'intérieur d'un paysage intellectuel français polarisé par une tension entre «nationalistes» et «cosmopolites» à la fin du XIX siècle, pour reprendre la dichotomie proposée par Blaise Wilfert-Portal (2016), les promoteurs des langues vivantes utilisent des arguments issus des deux sphères antagonistes. Bien que leur audience sur la scène intellectuelle reste modeste, ils n'en cherchent pas moins à s'adresser à un public allant au-delà des seuls pédagogues et spécialistes. À ce titre, ils peuvent être définis comme des intermédiaires culturels non seulement entre leur pays et les aires géographiques dont ils enseignent la langue, mais aussi entre des conceptions divergentes de la nation en France.

En termes d'histoire de l'éducation, dans le rapport qu'elles entretiennent avec les pays étrangers, les disciplines linguistiques apparaissent bien politiques chacune à leur façon. L'idée qu'il existerait en la matière une exception allemande résulte en bonne partie d'un effet de prisme, lié au surcroît de visibilité apporté par le grand nombre d'enseignants de cette langue dans les établissements secondaires et supérieurs, qui renforce la capacité de la discipline à produire des élites visibles dans les échanges entre les deux pays comme sur la scène nationale. À des échelles numériquement moins importantes, les enjeux associés à l'enseignement du russe ou de l'italien apparaissent finalement tout autant englobés dans des dynamiques qui font de ces savoirs scolaires des objets de transaction dans les relations internationales. L'échec de mécanismes de transferts, comme celui de l'enseignement du russe en lycée en France au début des années 1900, n'en diminue en rien l'intérêt historique rétrospectif. Les professeurs de langue vivante, dès lors, apparaissent bien comme des acteurs sinon directement «engagés» au 
sens donné classiquement à l'intervention d'intellectuels dans le débat public depuis l'affaire Dreyfus, du moins comme des acteurs impliqués dans le système international. La question des usages politiques et sociaux de l'enseignement des langues vivantes

\section{Bibliographie}

BENNASSAR B. (1994). «Panorama de I'hispanisme français ». In J. Sagnes, Images et influences de l'Espagne dans la France contemporaine. Perpignan : Presses universitaires de Perpignan, p.27-33.

BERGÈRE M.-C. \& PINO A. (1995). Un siècle d'enseignement du chinois à l'École des langues orientales. 1840-1945: bicentenaire des Langues orientales. Paris : L'Asiathèque.

BRETHOMÉ J. (2004). La langue de l'autre : histoire des professeurs d'allemand des lycées, 1850-1880. Grenoble : ELLUG.

BOURLET M. (2007) «Des normaliens dans les services de renseignement du ministère de la guerre (1914-1918)». Revue historique des armées, $\mathrm{n}^{\circ} 247, \mathrm{p} .31-41$.

CARDON-QUINT C., D'ENFERT R. \& PICARD E. (2014) «Les associations de spécialistes : militantisme et identités professionnelles ( $\mathrm{XX}^{\mathrm{e}}-\mathrm{XXI}{ }^{\mathrm{e}}$ siècle) ». Histoire de l'éducation, $n^{\circ} 142$.

CHANET J.-F. (1996). L'école républicaine et les petites patries. Paris : Aubier.

CHARLE C. (1994). «Ambassadeurs ou chercheurs? Les relations internationales des professeurs de la Sorbonne sous la III République». Genèses, n 14, p. 42-62.

CHAUBET F. (2006). La politique culturelle française et la diplomatie de la langue : I'Alliance française, 1883-1940. Paris : L'Harmattan.

CHERVEL A. (1993). Histoire de l'agrégation : contribution à I'histoire de la culture scolaire. Paris : INRP \& Kimé.

CHERVEL A. (2015). Les agrégés de l'enseignement secondaire. Répertoire 1809-1960. En ligne: <http://rhe.ish-lyon.cnrs. $\mathrm{fr} / \mathrm{q} \mathrm{q}=$ agregsecondaire_laureats $>$ (consulté le 30 mars 2017).

CCEURÉ S. (2004). «La langue russe et la "carte mentale" de I'Europe au XX $X^{e}$ siècle. Réflexions sur l'exemple français ». Matériaux pour l'histoire de notre temps, $\mathrm{n}^{\circ} 76, \mathrm{p} .27-33$.

CONDETTE J.-F. (2006a). Les lettrés de la République. Villeneuve-d'Ascq : Institut de Recherches historiques du Septentrion.

CONDETTE J.-F. (2006b). Les recteurs d'académie en France de 1808 à 1940. Lyon : INRP, Service d'histoire de l'éducation.

CONDETTE J.-F. (2009). Albert Châtelet. La République par l'école (1883-1960). Arras : Artois Presses Universités.

CONDETTE J.-F. (2015). Les chefs d'établissement. Diriger une institution scolaire ou universitaire, $X V I I^{e}-X X^{e}$ siècle. Rennes: Presses universitaires de Rennes.

DANIEL Y., GRANGÉ P., ZHUXIANG H. et al. (2015). FranceChine: les échanges culturels et linguistiques, histoire, appelle dès lors sans doute encore d'importantes recherches individuelles et collectives.

Jérémie Dubois

Université de Reims, laboratoire CERHiC (EA 2616) jeremie.dubois@univ-reims.fr

enjeux, perspectives. Rennes : Presses universitaires de Rennes.

DAVID M. (1984). «Pour une chronographie des études italiennes en France». Revue des études italiennes, n³0, p. 129-165.

DEJOB C. (1919). Histoire de la Société d'études italiennes. Paris : de Boccard.

DELAUNAY (1994). Des palais en Espagne: I'École des hautes études hispaniques et la Casa de Velázquez au coeur des relations franco-espagnoles du XXe siècle, 1898-1979. Madrid : Casa de Velázquez.

DELAUNAY J.-M. (2010). Méfiance cordiale. Les relations franco-espagnoles de la fin du XIXe siècle à la Première Guerre mondiale. Vol. 1, Les relations métropolitaines. Paris : L'Harmattan.

DRIJARD A. (1960). «Nécrologies. Joseph Denis 1882-1960». Les Langues modernes, $54^{\mathrm{e}}$ année, $\mathrm{n}^{\circ} 4$, p. 9-10.

DUBOIS J. (2015a). L'enseignement de l'italien en France (18801940). Une discipline au cour des relations franco-italiennes. Grenoble : ELLUG.

DUBOIS J. (2015b). «Les associations de professeurs d'italien en France. Militantisme disciplinaire et stratégies d'influence transnationales de la fin du XIX ${ }^{\mathrm{e}}$ siècle aux lendemains de la Première Guerre mondiale». Laboratoire italien, $\mathrm{n}^{\circ} 16$. En ligne : <https://journals.openedition.org/ laboratoireitalien/946> (consulté le 30 mars 2017).

DUBOIS J. (2015c) «Les lecteurs d'italien dans les universités françaises pendant le fascisme: des stratégies contrariées d'influence par la langue». Mélanges de l'École française de Rome - Italie et Méditerranée modernes et contemporaines, $\mathrm{n}^{\circ} 127-2$. En ligne: <http://journals.openedition.org/ mefrim/2286> (consulté le 8 juin 2018).

EFTHYMIOU L. (2003). «Le genre des concours». Clio, Histoire, femmes et sociétés, $\mathrm{n}^{\circ} 18, \mathrm{p} .91-112$.

ESPAGNE M. (1993). Le paradigme de l'étranger. Les chaires de littérature étrangère au XIX siècle. Paris : Cerf.

ESPAGNE M. \& WERNER M. (1994). Histoire des études germaniques en France : 1900-1970. Paris : CNRS.

ESPAGNE M., LAGIER, F. \& WERNER M. (1991). Le Maître de langues. Les premiers enseignants d'allemand en France (18301850). Paris : Édition de la Maison des sciences de I'homme.

GERMAIN C. (1993). Évolution de l'enseignement des langues: 5000 ans d'histoire. Paris : Clé International.

GESLOT J.-C. (2009). Victor Duruy, historien et ministre, 18111894. Villeneuve-d'Ascq : Presses universitaires du Septentrion. 
GUICHARD B. (1999). «Parler russe, un enjeu de I'alliance franco-russe ». Bulletin de I'Institut Pierre Renouvin, $\mathrm{n}^{\circ} 8$, p. 119-137.

HERRERAS J. C. (2008). «La place de l'espagnol dans l'enseignement secondaire et supérieur en France ». In J. C. Herreras, L'enseignement de l'espagnol en France : réalités et perspectives. Valenciennes: Presses universitaires de Valenciennes, p.11-26.

HÉRY E. (2015). «1902 : retour sur la réforme de l'enseignement secondaire». Le Débat, vol.5, n¹87, p.169-177.

ISAMBERT-JAMATIV. (1969). «Une réforme des lycées et collèges. Essai d'analyse sociologique de la réforme de 1902 ». L'Année sociologique, $3^{\mathrm{e}}$ série, $\mathrm{n}^{\circ} 20$, p. 9-60.

LOUBES O. (2012). Jean Zay: I'inconnu de la République. Paris : Armand Colin.

LUCHAIRE J. (1902). «Leçon d'ouverture du cours de langue et de littérature italiennes à la faculté des Lettres de I'Université de Lyon ». Revue internationale de l'enseignement, $n^{\circ} 43$, p. 259-273.

MESSAOUDI A. (2008). «Origines et enjeux initiaux de l'agrégation d'arabe». In Le centenaire de l'agrégation d'arabe, actes du colloque organisé par la Direction générale de I'Enseignement scolaire, Institut du monde arabe/La Sorbonne, les 17 et 18 novembre 2006. En ligne : <http:// media.eduscol.education.fr/file/Formation_continue_ enseignants/19/7/actes_agregation_arabe_110197.pdf> (consulté le 30 mars 2017).

MESSAOUDI A. (2011). «Des médiateurs effacés? Les professeurs d'arabe des collèges et lycées d'Algérie (1840-1940)». Outre-mers, t. 98, n³70-371, p.149-159.

MESSAOUDI A. (2013). «Progrès de la science, développement de l'enseignement secondaire et affirmation d'une "méthode directe" (1871-1930)». In S. Larzul \& A. Messaoudi, Manuels d'arabe d'hier et d'aujourd'hui, XIXe$X X I^{e}$ siècle. Paris : Bibliothèque nationale de France.

MESSAOUDI A. (2015). Les arabisants et la France coloniale. Savants, conseillers, médiateurs (1780-1930). Lyon : ENS Éditions.

MICHEL Y. (2016). «Compromis plutôt que réforme : la constitution des langues régionales comme discipline scolaire au XXe siècle». In P. Kahn \& Y. Michel, Formation, transformations des savoirs scolaires. Histoires croisées des disciplines, $X I X^{e}-X X^{e}$ siècles. Caen : Presses universitaires de Caen.

MOMBERT M. (2001). L'enseignement de l'allemand en France, 1880-1918. Entre "modèle allemand» et "langue de l'ennemi». Strasbourg : Presses universitaires de Strasbourg.

MOMBERT M. (2005). «De crise en crise : l'enseignement de l'allemand de 1918 à 1939 ». Histoire de l'éducation, n 106, p.71-136.

MOMBERT M. (2014). "Les revues de langues vivantes en France et en Allemagne autour de 1900 : la fabrique d'une opinion publique enseignante». Thema. Revue suisse des sciences de l'éducation, n³6-1, p. 35-49.

NINO RODRIGUEZ A. (1988). Cultura y diplomacia : los hispa- nistas franceses y España de 1875 a 1931. Madrid : Casa de Velàzquez/Société des hispanistes français.

NOIRIEL G. (2010). Le massacre des Italiens : Aigues-Mortes, 17 août 1893. Paris : Fayard.

POULY M.-P. (2007). «La promotion d'un savoir utile dans l'ordre scolaire français de la Troisième République : le cas des langues étrangères ». Cahiers de la recherche sur l'éducation et les savoirs, n6, p. 119-134.

POULY M.-P. (2009). L'«esprit» du capitalisme et le corps des lettrés. L'inscription scolaire de l'anglais et sa différenciation, $X I X^{e}-X X^{e}$ siècles. Thèse de doctorat, sociologie, EHESS.

POULY M.-P. (2010). "La définition pratique des langues vivantes dans la réforme du baccalauréat de $1902 »$. In P. Marchand, Le baccalauréat (1808-2006). Certification française ou pratique européenne. Lyon/Villeneuve-d'Ascq : INRP/Revue du Nord, p.265-277.

POULY M.-P. (2012). «La différenciation sociale de l'apprentissage de la langue anglaise en France au XIX siècle». Histoire de l'éducation, n¹33, p. 5-41.

PROST A. (2008). «De l'enquête à la réforme. L'enseignement secondaire des garçons de 1898 à $1902 »$. Histoire de l'éducation, $\mathrm{n}^{\circ} 119$, p.29-80.

PUREN C. (1988). Histoire des méthodologies de l'enseignement des langues. Paris : Clé International.

RADTKA C. (2013). Construire la société scientifique par l'école. Angleterre, France et Pologne au prisme des manuels de sciences pour les élèves ordinaires (1950-2000). Thèse de doctorat, histoire des sciences, EHESS.

RADTKA C. (2018). «De la construction des nations modernes à un repli sur le présent. Le cas des manuels de sciences français, polonais et anglais entre les années 1950 et les années 2000 ». In J. Dubois \& P. Legris, Disciplines scolaires et cultures politiques. Des modèles nationaux en mutation depuis 1945. Rennes: Presses universitaires de Rennes, p. 185-200.

RIVAL S. (2012). L'échange des assistants de langue vivante entre la France et l'Allemagne avant la Seconde Guerre mondiale: les «directeurs de conversation » et la «langue de l'ennemi ». Thèse de doctorat, études germaniques, université de Lorraine.

ROGERS R. (2006). «Les femmes dans l'enseignement des langues vivantes : éléments pour une histoire à construire». Ela. Études de linguistique appliquée, vol.2, $\mathrm{n}^{\circ} 142$, p. 135-149.

TAUCH E. (1994). "Les échanges de lecteurs d'universités entre la France et l'Allemagne, des origines à 1939 ». In M. Espagne \& M. Werner, Histoire des études germaniques en France : 1900-1970. Paris : CNRS, p.307-320.

TRONCHET G. (2016). «L'ouverture internationale des universités en France (1860-1914) ». In C. Charle \& L. Jeanpierre, La vie intellectuelle en France. Volume I. Des lendemains de la Révolution à 1914. Paris : Éd. du Seuil, p.614-617.

VERNEUIL Y. (2005). Les agrégés. Histoire d'une exception française. Paris : Belin.

VERNEUIL Y. (2014). «Corporation universitaire et société 
civile : les débats sur la composition du Conseil supérieur de l'instruction publique pendant la Troisième République». Histoire de l'éducation, n`140-141, p.51-72.

WAQUET F. (1998). Le Latin ou l'empire d'un signe, XVI $X X e$ siècles. Paris : Albin Michel.
WILFERT-PORTAL B. (2016). «Une nouvelle géopolitique intellectuelle. Entre nationalisme et cosmopolitisme ». In C. Charle \& L. Jeanpierre, La vie intellectuelle en France. Vol. I. Des lendemains de la Révolution à 1914. Paris : Éd. du Seuil, p.559-591. 
